\title{
STUDY ON INFLUENCE OF BAIMAO SANDBANK CONTROL PROJECT IN SOUTHERN BRANCH OF THE YANGTZE RIVER ESTUARY, CHINA
}

\author{
Xinzhou Zhang ${ }^{1}$, Xiping Dou ${ }^{2}$ and Jinshan Zhang ${ }^{3}$
}

\begin{abstract}
The author establishes the Yangtze estuary 2D tidal and sediment mathematical model based on the orthogonal curvilinear coordinate system. The erosion and siltation of engineering section under the hydrological conditions of flood season, dry season and the great flood after the Baimao Sand control engineering is calculated. Preliminarily expounds the correlation of Baimao Sand and Biandan Sand and the impact of Biandan Sand after the Baimao Sand control engineering is implemented.
\end{abstract}

Keywords: the Baimao Sand; the Biandan Sand; mathematical model; impact analysis

\section{INTRODUCTIONS}

The Baimao Sand waterway is located on the upper ridge of the southern branch of the Yangtze River. The upper riverway is divided into southern and northern waterway of Baimao Sand because of the existence of the Baimao sand. The Baimao Sand waterway is located under the tidal current limit and influenced by the upstream runoff and downstream tidal. Being one of three main navigation waterways from the Nanjing to Liuhe mouth, the channel's maintenance and development is influenced by the complex flow conditions, shoal erosion and siltation, inlet rise and decline. As a result, control engineering is considered in the head Baimao Sand to stabilize the current good regime of Baimao Sand riverway. The Biandan Sand is a huge beach located on the southern shore of lower segment of southern branch. Bounded by the south gate port in Chongming, the west part is called upper Biandan Sand, the east part is called the lower Biandan Sand(Figure. 1). The upper Biandan Sand is the north boundary of lower segment of North Baimao Sand waterway, the lower Biandan Sand is the north shore wall of Sorth branch lower segment of Yangtze River's mainstream channel. The pattern and trend of Biandan Sand directly influences the trend of lower segment of Sorth branch of Yangtze River's mainstream channel. Hence, the research of the correlation of Baimao Sand and Biandan Sand and the impact of Biandan Sand after the Baimao Sand control engineering is implemented is needed.
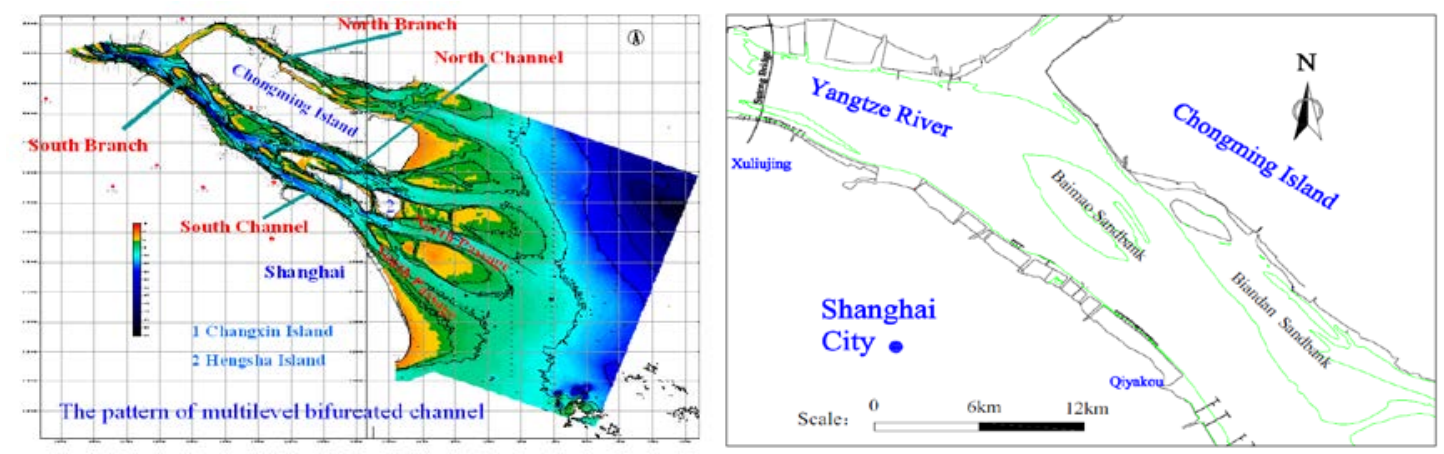

Figure. 1 the pattern of multilevel bifurcated channel

\section{TIDAL AND SEDIMENT MOVEMENT FUNDAMENTAL EQUATION}

Tidal Movement Fundamental Equation

Under the Cartesian coordinate system $\xi-\eta$, the 2D tidal movement fundamental equation is as follow:

\footnotetext{
${ }^{1}$ Nanjing Hydraulics Research Institute, Key Lab of Port, Waterway and Sedimentation Engineering of the Ministry of Transport, 223 Guangzhou Road, Nanjing, Jiangsu, 210029, China

${ }^{2}$ Nanjing Hydraulics Research Institute, Key Lab of Port, Waterway and Sedimentation Engineering of the Ministry of Transport, 223 Guangzhou Road, Nanjing, Jiangsu, 210029, China

${ }^{3}$ Nanjing Hydraulics Research Institute, Key Lab of Port, Waterway and Sedimentation Engineering of the Ministry of Transport, 223 Guangzhou Road, Nanjing, Jiangsu, 210029, China
} 


$$
\begin{gathered}
\frac{\partial \varsigma}{\partial t}+\frac{1}{g_{\xi} g_{\eta}} \frac{\partial}{\partial \xi}\left(H_{u g_{\eta}}\right)+\frac{1}{g_{\xi} \boldsymbol{g}_{\eta}} \frac{\partial}{\partial \eta}\left(\mathrm{Hvg}_{\xi}\right)=0 \\
\frac{\partial u}{\partial t}+\frac{u}{g_{\xi}} \frac{\partial u}{\partial \xi}+\frac{v}{g_{\eta}} \frac{\partial u}{\partial \eta}+\frac{u v}{g_{\xi} g_{\eta}} \frac{\partial g_{\xi}}{\partial \eta}-\frac{v^{2}}{g_{\xi} g_{\eta}} \frac{\partial g_{\eta}}{\partial \xi}+g \frac{u \sqrt{u^{2}+v^{2}}}{C^{2} H}-f v+\frac{g}{g_{\xi}} \frac{\partial \varsigma}{\partial \xi}=E\left(\frac{1}{g_{\xi}} \frac{\partial A}{\partial \xi}-\frac{1}{g_{\eta}} \frac{\partial B}{\partial \eta}\right) \\
\frac{\partial v}{\partial t}+\frac{u}{g_{\xi}} \frac{\partial v}{\partial \xi}+\frac{v}{g_{\eta}} \frac{\partial v}{\partial \eta}+\frac{u v}{g_{\xi} g_{\eta}} \frac{\partial g_{\eta}}{\partial \xi}-\frac{u^{2}}{g_{\xi} g_{\eta}} \frac{\partial g_{\eta}}{\partial \xi}+g \frac{v \sqrt{u^{2}+v^{2}}}{C^{2} H}+f u+\frac{g}{g_{\eta}} \frac{\partial \varsigma}{\partial \eta}=E\left(\frac{1}{g_{\xi}} \frac{\partial B}{\partial \xi}+\frac{1}{g_{\eta}} \frac{\partial A}{\partial \eta}\right)
\end{gathered}
$$

Where,

$$
A=\left[\frac{\partial}{\partial \xi}\left(u g_{\eta}\right)+\frac{\partial}{\partial \eta}\left(v g_{\xi}\right)\right] / g_{\xi} g_{\eta} ; B=\left[\frac{\partial}{\partial \xi}\left(v g_{\eta}\right)-\frac{\partial}{\partial \eta}\left(u g_{\xi}\right)\right] / g_{\xi} g_{\eta} ; g_{\xi}=\sqrt{x_{\xi}^{2}+y_{\xi}^{2}} ; g_{\eta}=\sqrt{x_{\eta}^{2}+y_{\eta}^{2}} \text {. }
$$

$u$ and $v$ are the flow velocity components on the direction of $\xi-\eta$; $\xi$ is water lever, $H$ is total water depth; $C$ is Chezy coefficient, $g_{\xi}, g_{\eta}$ are Lami coefficient; $E$ is viscosity coefficient of flow turbulence.

\section{Sediment Movement Fundamental Equation}

Under the Cartesian coordinate system, Dou Guoren 2d suspended sediment transport equation is as follow:

$$
\frac{\partial(H S)}{\partial t}+\frac{1}{g_{\xi} g_{\eta}}\left[\frac{\partial\left(H u S g_{\xi}\right)}{\partial \xi}+\frac{\partial\left(H v S g_{\xi}\right)}{\partial \eta}\right]+\alpha \omega\left(S-S_{*}\right)=0
$$

Where $S$ is vertical average sediment concentration; $\alpha$ is an undetermined coefficient and is defined by validation calculation; $\omega$ is sediment settling velocity, and equals to flocculation settling velocity under the flocculation condition; $S_{*}$ is sediment carrying capability of the flow, with the Dou Guoren formula, can be expressed under the combined action of tidal and wave as follow:

$$
S_{*}=\alpha_{0} \frac{\gamma \gamma_{s}}{\gamma_{s}-\gamma}\left[\frac{\left(u^{2}+v^{2}\right)^{3 / 2}}{C^{2} H \omega}+\beta_{0} \frac{H_{W}{ }^{2}}{H T \omega}\right]
$$

Where $\gamma$ and $\gamma_{S}$ are volume-weight of water and sediment particle, $H_{w}$ and $T$ are average wave height and period. According to the data obtained in many sea areas, $\alpha_{0}=0.023, \beta_{0}=0.04 f_{w}$, $f_{w}$ is wave drag coefficient. Chezy coefficient is defined by Manning formula, $C=(1 / n) H^{1 / 6}, n$ is the surface roughness coefficient.

Bed Load Sediment Transport Fundamental Equation

Dou Guoren bed load unbalance sediment transport fundamental equation is as follow:

$$
\frac{\partial(H N)}{\partial t}+\frac{\partial(H N u)}{\partial x}+\frac{\partial(H N v)}{\partial y}=\alpha_{b} \omega_{b}\left(N-N^{*}\right)
$$

Where $N$ is bed load sediment concentration in unit volume; $\alpha_{b}$ is bed load sedimentation coefficient, $\omega_{b}$ is bed load particle settling velocity; $N^{*}$ is defined as follow:

$$
N^{*}=q_{b}^{*} / H v
$$

Where $q_{b}^{*}$ is bed load sediment transport ability in the condition of unit time and unit width, defined by Dou Guoren formula. 
Riverbed Deformation Fundamental Equation

Riverbed deformation equation caused by suspended load is as follow:

$$
\gamma_{0} \frac{\partial \eta_{S}}{\partial t}=\alpha \omega\left(S-S_{*}\right)
$$

Where $\eta_{s}$ is silting and scouring thickness caused by suspended load.

Riverbed deformation equation caused by bed load is as follow:

$$
\gamma_{0} \frac{\partial \eta_{b}}{\partial t}=\alpha_{b} \omega_{b}\left(N-N^{*}\right)
$$

Where $\eta_{b}$ is silting and scouring thickness caused by bed load.

Silting and scouring thickness caused by suspended load and bed load is as follow:

$$
\eta_{S}=\eta_{S}+\eta_{b}
$$

\section{THE CALIBRATION AND VALIDATION OF TIDAL AND SEDIMENT MATHEMATICAL MODEL}

\section{The Calculation Area and Calculation Grid}

According to the research contents and mission requirements, and in order to satisfy the water flow movement similarity, this mathematical model calculation scope is west to Jiangyin, east to $123^{\circ}$ east longitude , south to $29^{\circ} 27^{\prime}$ north latitude, north to $32^{\circ} 15^{\prime}$ north latitude, including the waters of the Yangtze estuary and the Hangzhou bay model. Figure 2 is the calculation scope of the mathematical model. Because of the shape of Yangtze estuary boundary is very complex, the model uses orthogonal curvilinear grid which fits the boundary of the river as the computing grid, and refines the grid in engineering section, the grid number is $486 \times 346=168156$, the scale is between $10 \sim 2000 \mathrm{~m}$. Figure 3 is the calculation grid of the mathematical model.

Figure 2. the calculation scope of the mathematical model.
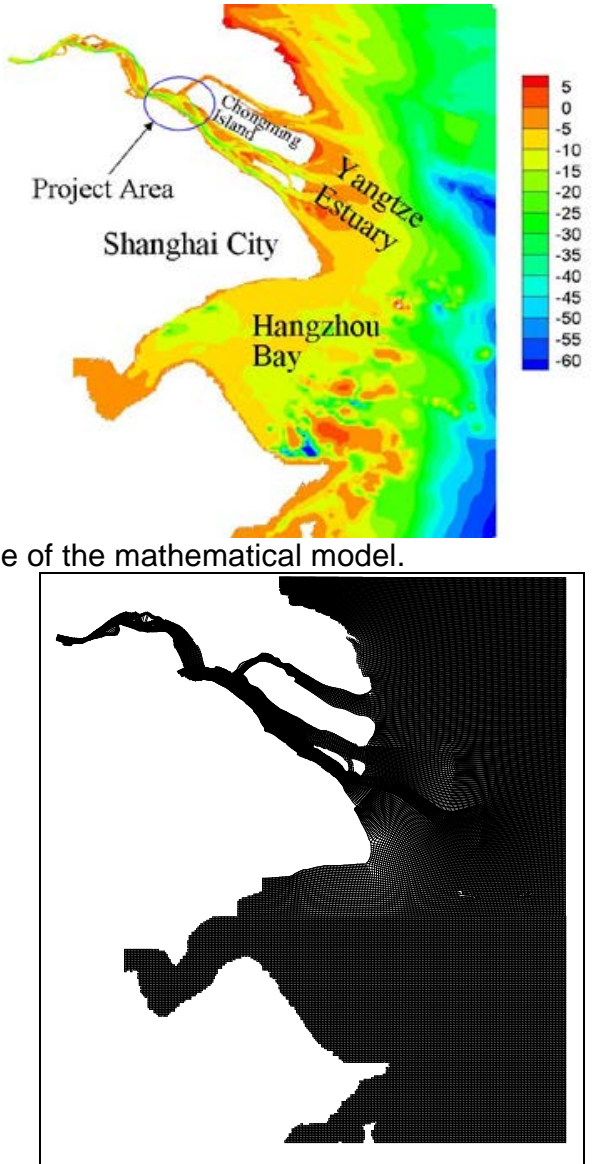

Figure 3. the calculation grid of the mathematical model. 


\section{Calibration and Validation of Tidal Mathematical Model}

The control tide level of the open boundary is provided by Hohai University calculating the east China sea mock- up model, the upstream open boundary is provided by the Yangtze river scientific research institute of the Changjiang Water Resources Committee calculating the Jiangyin flow process.

The upstream sediment concentration hygrograph of the open boundary is calculated by the 1d tidal and sediment mathematical model, the sediment concentration of the off-sea boundary is $0.1 \mathrm{~kg} / \mathrm{m}^{3}$.Using the excess water depth method, continuously fixing water boundaries waterfront and automatically recognizes the boundary according to the change of water level. When the upstream and off-sea boundary is given, parameters debuged through calibration, according to the linear interpolation, the roughness from coast to upstream is defined as $n=0.012+0.01 / H \sim 0.02+0.01 / H$, turbulence viscous coefficient $v_{t}$ is $30 \mathrm{~m}^{2} / \mathrm{s}$, the computation time step of tide mathematical model is 4 seconds. The time step of total load mathematical model is 20 seconds, the roughness is the same as the tide mathematical model. The sediment particle volume-weight $\gamma_{s}=2650 \mathrm{~kg} / \mathrm{m}^{3}$, suspended load dry volume-weight is $\gamma_{s}=750 \mathrm{~kg} / \mathrm{m}^{3}$, the bed load dry volume-weight is $\gamma_{o b}=1200 \mathrm{~kg} / \mathrm{m}^{3}$. The sediment particle of the upper Gaoqiao water area is not flocculated, considered as $d_{50}=0.01 \mathrm{~mm}$, water temperature is high in the flood season, settling velocity $\omega=0.0001 \mathrm{~m} / \mathrm{s}$; water temperature is low in the dry season, settling velocity $\omega=0.00008 \mathrm{~m} / \mathrm{s}$. The sediment particle settling velocity of the lower Gaoqiao water area differs with the changed salinity and temperature in the flood and dry season, hence the settling velocity of suspended particle is generalized to $0.00008 \mathrm{~m} / \mathrm{s} \sim 0.0007 \mathrm{~m} / \mathrm{s}$. The bed load sediment particles $d_{50}=0.1 \mathrm{~mm}$ on the beach face, $d_{50}=0.14 \mathrm{~mm}$ at the bottom of slot.

\section{ENGINEERING PLAN AND TIDAL CALCULATION AND THE ANALYSIS OF THE RESULTS}

According to the arrangement of engineering plan, with the calculating conditions of spring tide of flood and dry season and 98 great flood, the engineering region terrain is latest measured in March 2010, using the validated and calibrated Yangtze estuary tidal mathematical model, researches the flow field changes before and after Baimao sand engineering plan is implemented, analyses the engineering plan's impact on the Biandan sand.

\section{Project Scheme}

According to the main problems of Baimao sand waterway, combined with the future developing trend, the regulation project layout plans of Baimao Sand waterway are defined, including the recommended plan (Scheme I), the elected plan(Scheme II) and the added plan(Scheme III). Three layout plans are as follow:

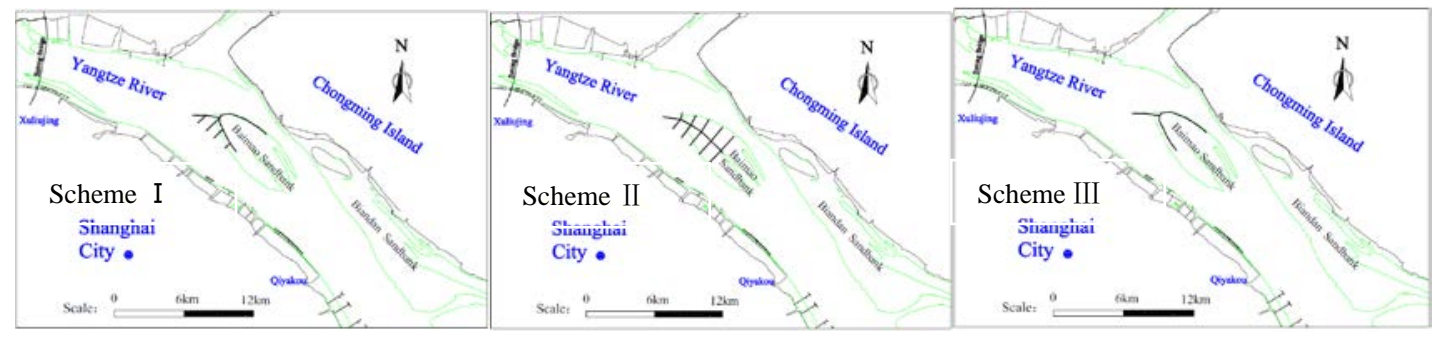

Figure 4. The schemes of Baimao Sandbank Control Project.

\section{Sediment Calculation and Analysis}

Sediment calculation conditions. According to the engineering project arrangement, uses hydrologic condition of flood season, dry season and the 98 great flood as calculation condition, researches the silting and scouring variation of this stream segment. The calculating groups are shown in table 1. 


\begin{tabular}{|l|l|l|}
\hline \multicolumn{2}{|l|}{ Table 1 The calculating groups } \\
\hline Project & Research section & Calculation hydrological conditions \\
\hline Scheme I & Baimao Sand-Biandan Sand & One month of flood season, dry season, great flood \\
\hline Scheme II & Baimao Sand-Biandan Sand & One month of flood season, dry season, great flood \\
\hline Scheme III & Baimao Sand-Biandan Sand & One month of flood season, dry season, great flood \\
\hline
\end{tabular}

Analysis of the results. After the project is implemented, the changes of hydrodynamic conditions lead to the corresponding changes of river bed's silting and scouring. In order to analysis the impact on the changes of the river bed's silting and scouring after each engineering plan is implemented, the impact of project on the silting and scouring of the stream segment is got after using the calculation results of each plan's silting and scouring minus the ones before the project is implemented.

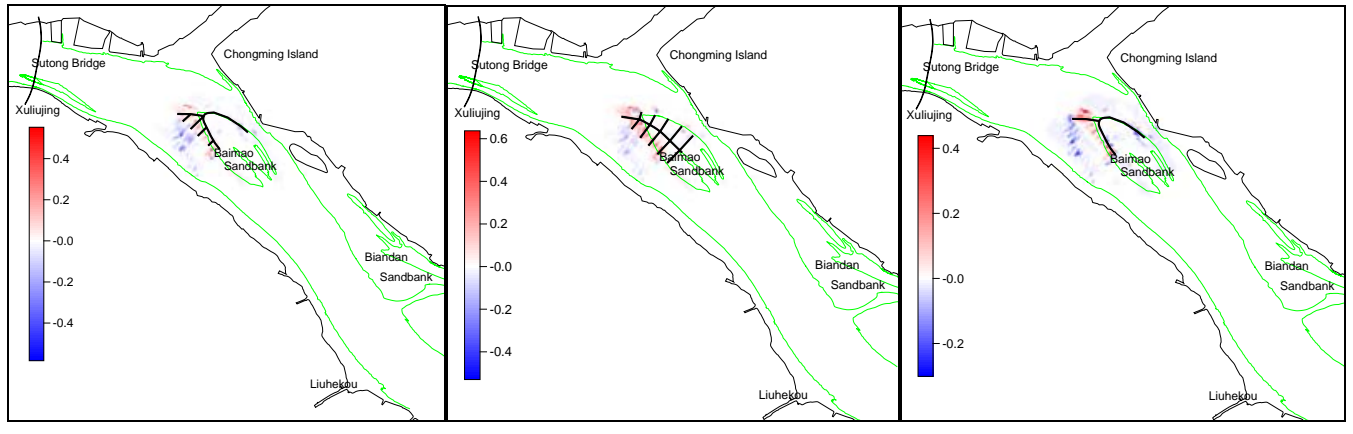

Figure5. Topography change after the implementation of Control Project (flood season) .

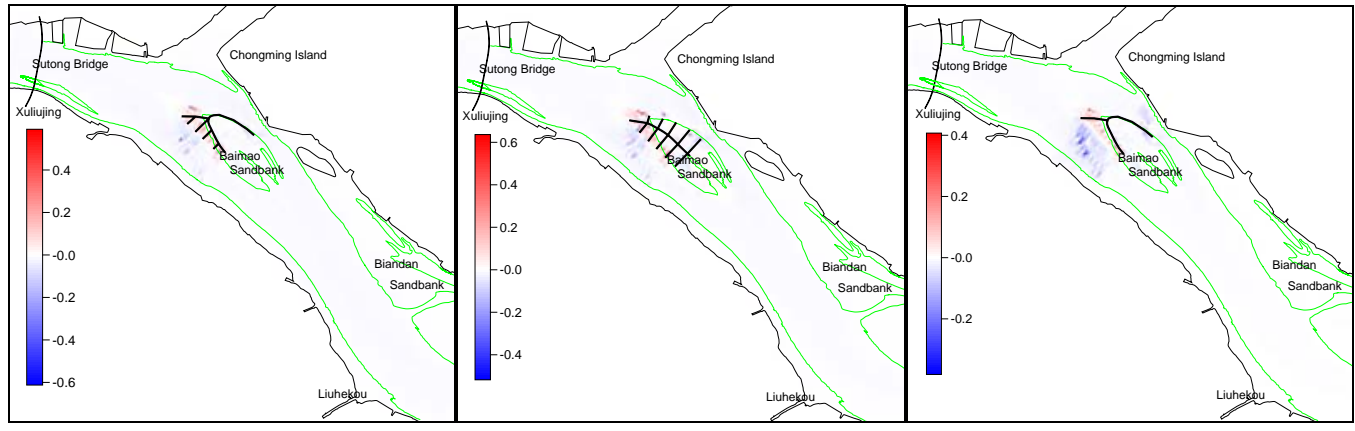

Figure6. Topography change after the implementation of Control Project (dry season) .

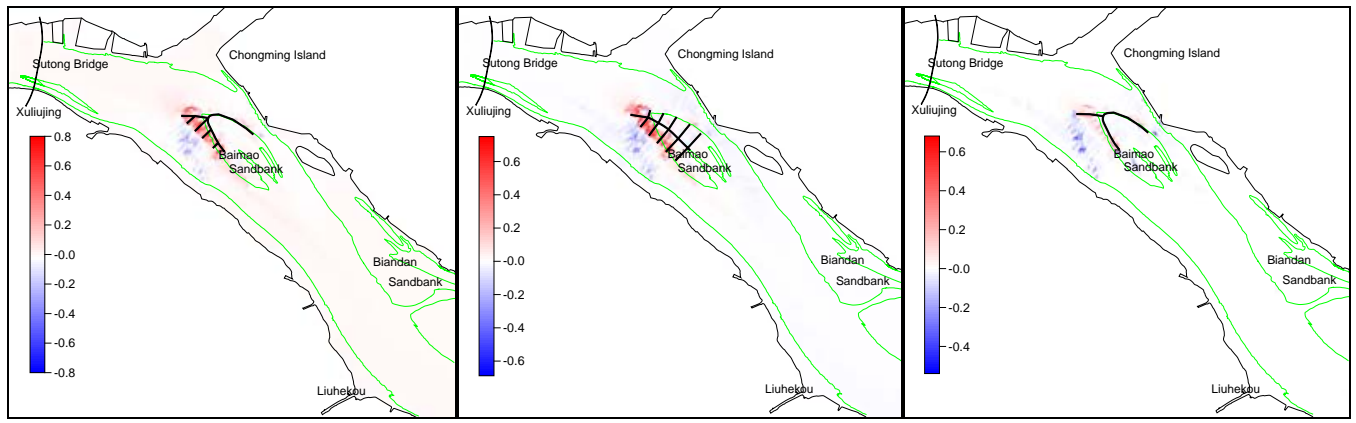

Figure7. Topography change after the implementation of Control Project (great flood) . 
The calculation results show that the project has limited influence on the silting and scouring range of the river bed and almost no influence on the river bed near the Biandan Sand after the engineering plan is implemented. The silting and scouring caused by the project is almost within $0.7 \mathrm{~m}$.

\section{CONCLUSION}

The preliminary conclusion is got through the research of Yangtze estuary 2D tidal sediment mathematical model as follow:

1. For the last 20 years, influenced by continuous floods from the Yangtze River, Baimao sandbank has tended to move down year by year and the area of Baimao sandbank body has become less and less. Therefore, control works shall be undertaken immediately to protect and maintain Baimao sandbank. To maintain the stability of Biandan sandbank body is important to keep the stability of the good river pattern on the lower side of the southern branch.

2. According to the calculations in one month flood season, dry season and flood, after the implementation of the control project, the scour and sedimentation in local parts of the project has relatively big changes which is basically within 1.0; it is slightly silted on Baimao sandbank sand body and the sheltered zone in the Yuguchi dam of Baimao sandbank control project is silted to $0.1 \sim 0.9 \mathrm{~m}$ thick, which is helpful for the stability of the pattern of Baimao sandbank sand body; for the southern channel and northern channel of Baimao sandbank, the upper side is scoured, the middle part is silted and the southern side of Baimao sandbank's end is scoured; Biandan sandbank is slightly scoured in its southern side and end of upper side with a scouring range within $0.55 \mathrm{~m}$ and $0.35 \mathrm{~m}$ respectively.

3. Analysis on the scour-and-fill of landform caused by one month flood season, dry season and flood and other conditions indicates that the implementation of all planning has limited influence on the riverbed in the matter of scour-and-fill which is mainly concentrated in the project areas with a range within $0.7 \mathrm{~m}$, basically having no impact on the scour-and-fill of the riverbeds near Biandan sandbank.

The engineering plan will not have an obvious impact on Biandan sand after the project is implemented through comprehensive analysis of flow field and sediment erosion and deposition changes

\section{ACKNOWLEDGMENTS}

The research work is supported by the Public Welfare Scientific Research Project of Ministry of Water Resources No. 200801016 and National Natural Science Foundation No. 51079088.

\section{REFERENCES}

Xia Yimin,Yuan Wenzhi(1998): Study on regulation works of the Xuliujing-Baimao sandbank segment of the Yangtze estuary, Ocean Engineering,Vol.16 No4, pp.93-103. 